\title{
Lidil
}

Revue de linguistique et de didactique des langues

$54 \mid 2016$

La phrase en production d'écrits, approches nouvelles en didactique

\section{La phrase à l'épreuve des textes scolaires (élèves de 5 à 8 ans)}

The Sentence Inside the Texts of Students from 5 to 8 Years

\section{Marie-Noëlle Roubaud}

\section{OpenEdition}

\section{Journals}

Édition électronique

URL : http://journals.openedition.org/lidil/4065

DOI : $10.4000 /$ lidil.4065

ISSN : 1960-6052

Éditeur

UGA Éditions/Université Grenoble Alpes

Édition imprimée

Date de publication : 20 novembre 2016

Pagination : 93-114

ISBN : 978-2-84310-339-1

ISSN : 1146-6480

Référence électronique

Marie-Noëlle Roubaud, « La phrase à l'épreuve des textes scolaires (élèves de 5 à 8 ans) », Lidil [En ligne], 54 | 2016, mis en ligne le 01 janvier 2017, consulté le 10 décembre 2020. URL : http:// journals.openedition.org/lidil/4065; DOI : https://doi.org/10.4000/lidil.4065 


\title{
La phrase à l'épreuve des textes scolaires (élèves de 5 à 8 ans)
}

\author{
Marie-Noëlle Roubaud*
}

\begin{abstract}
RÉSUMÉ
L'analyse linguistique des textes scolaires de jeunes élèves d'école primaire (5 à 8 ans), dans la perspective grammairienne des travaux du Groupe aixois de recherches en syntaxe (GARS), amène à repenser la notion de phrase alors que les préconisations institutionnelles (de 2008 en France) suggèrent de passer de l'écriture d'une phrase à celle de plusieurs phrases puis à celle d'un texte. Dans une première partie, nous reviendrons sur la notion de phrase à l'oral et à l'écrit. Nous mettrons en regard des travaux scientifiques et les résultats d'expérimentations concernant les représentations d'enfants de 6 à 8 ans. Dans une deuxième partie, l'expertise de productions d'élèves de 5 à 8 ans identifiera dans un premier temps le traitement d'unités syntaxiques par ces jeunes scripteurs et dans un second temps quelques conduites discursives que l'utilisation du point autorise.
\end{abstract}

\section{ABSTRACT}

The linguistic analysis of texts written by young school children (5-8 years old), in the framework of the Groupe Aixois de Recherches en Syntaxe (GARS), requires to reconsider the notion of sentence while the institutional recommendations (2008 in France) suggest to lead the children to write isolated sentences in the first place, several sentences in a second stage, and finally a whole text. In the first part of our paper, we will deal with the notion of sentence in spoken and written language. We will confront our descriptive framework with the results of tests concerning the representations of children aged from 6 to 8 . In the second part, the expertise of productions of children from 5 to 8 years of age will initially identify the treatment of syntactic units by these young writers and then will lead to some remarks about some discursive conducts allowed by the use of full-stop.

* Aix-Marseille Université, ADEF. 


\section{Introduction}

Les programmes ministériels français de $2008^{1}$ stipulent que, pour écrire un texte, l'élève doit d'abord "écrire de manière autonome une phrase simple cohérente, puis plusieurs, puis un texte narratif ou explicatif de 5 à 10 lignes » (p. 31). La hiérarchie des unités proposées (une phrase, plusieurs phrases, un texte) présuppose qu'une production écrite n'est qu'une suite de phrases. Mais de quelle phrase s'agit-il?

Dans une première partie, nous reviendrons sur la notion de phrase à l'oral et à l'écrit et nous la mettrons en regard des représentations que des élèves de 6 à 8 ans ont sur la phrase; nous identifierons ainsi des repères sur lesquels ces jeunes élèves s'appuient au moment d'identifier une phrase ou de segmenter un texte en phrases.

Puis, dans une seconde partie, nous examinerons la façon dont des élèves de 5 à 8 ans mettent à l'épreuve la phrase dans leurs productions. Nous nous appuierons sur un échantillon de textes représentatifs des manières de faire récurrentes d'élèves jeunes pour segmenter leur texte. L'analyse qualitative de ces écrits illustrera la mise en application de certains repères précédemment identifiés. Elle montrera que les jeunes scripteurs privilégient les unités syntaxiques aux unités graphiques. Mais elle nous fera également découvrir que la phrase est à envisager dans son rapport avec le texte : la non-conformité du découpage phrastique cache en effet des modes inédits d'organisation discursive qui dépassent le niveau des unités syntaxiques pour envisager celui des unités textuelles.

\section{La notion de phrase}

\subsection{Distorsion entre la langue orale et la langue écrite}

La notion de phrase n'a de réalité qu'à l'écrit, elle n'est pas dans la compétence des locuteurs qui ne peuvent s'appuyer sur l'oral pour la construire (Avanzi, 2007; Blanche-Benveniste, 1993). En effet, les pauses à l'oral ne marquent pas la limite des unités syntaxiques (Blanche-Benveniste, Bilger, Rouget \& Eynde, 1990); elles apparaissent parfois dans des positions où les éléments sont syntaxiquement très liés, par exemple entre le déterminant et le nom (ex.1) ou entre le groupe occupant la fonction de sujet et le verbe de la phrase (ex. 2) :

1. Programmes parus au Bulletin officiel $(B O)$ en vigueur au moment de l'écriture de l'article. 
(1) il ne me manquait + que le + violon $^{2}$ (p. 243).

(2) et ces assemblées d'actionnaires font d'ailleurs l'objet de procès verbaux lesquels sont consignés dans les registres + dont je + t'ai parlé tout à l'heure (p. 251).

En réalité, la notion de phrase n'a de réalité qu'à l'écrit, comme le mentionne Blanche-Benveniste, dès 1979, dans un manuscrit (Roubaud, 2013, p. 26) :

On ne peut pas, pour l'enseigner [la phrase], s'appuyer sur une connaissance naturelle de celui qui ne sait pas encore écrire. Il n'a rien en tête qui corresponde à phrase. C'est à force d'en entendre traiter, par une suite d'essais et d'erreurs dans la pratique, qu'il parviendra à y croire. Difficulté de l'enseignement de la ponctuation!

Or la phrase est une unité de lecture indispensable en classe pour l'apprentissage de la lecture (Gomila, 2009, p. 95). En tant que phrase graphique, elle représente une unité textuelle identifiable par les élèves, la ponctuation étant un auxiliaire précieux de la compréhension (Campana, 2002, p. 53). Par la lecture à haute voix d'un texte dès le cours préparatoire (à 6 ans), l'enseignant invite l'élève à respecter le découpage indiqué par les signes de ponctuation et à considérer certains signes comme des marqueurs de pause ou d'intonation qui aident à la lecture.

Pour les enseignants, le mot «phrase» est associé à sa représentation canonique, telle qu'elle s'est construite au cours du XVIII siècle (Seguin, 1993) en «sujet-verbe-objet» de sorte que l'école continue de véhiculer l'idée d'une coïncidence parfaite entre la phrase graphique et la phrase syntaxique pour d'évidentes raisons tenant à l'apprentissage de l'écrit. Ce savoir scolaire institutionnalisé par les manuels scolaires pour définir la phrase semble traverser le temps, comme dans la grammaire de Dubois et Lagane (1973) :

Qu'est-ce qu'une phrase?

Les énoncés sont formés de phrases. Les phrases sont des suites de mots ordonnés d'une certaine manière, qui entretiennent entre eux certaines relations, c'est-à-dire qui répondent à certaines règles de grammaire et qui ont un certain sens. Ainsi :

2. La transcription des exemples oraux s'appuie sur les conventions du Groupe aixois de recherches en syntaxe (GARS) : Équipe DELIC. (2004). Présentation du Corpus de référence du français parlé. Recherches sur le français parlé, 18, 11-42. 
Les nuages tombent dans le ciel.

est une phrase. (p. 14)

Chiss et David (2011) déclarent cependant que : «Sur le versant pédagogique par conséquent, la phrase du grammairien... n'existe simplement pas.» (p. 179) Il suffit de lire des textes d'auteurs pour le constater, comme le confirme Béguelin (2002) : «Caractéristiques d'une façon d'écrire actuellement à la mode, plusieurs des phrases graphiques [...] échappent ainsi aux normes de complétude sémanticogrammaticales communément associées à l'idée de phrase.» (p. 99). En voici un exemple (Doc. 1), extrait d'un album accompagnant une méthode de lecture pour des élèves de 6 ans (Un monde à lire, $\mathrm{CP}$, Nathan, 2005), qui prouve bien qu'on est loin de l'image de la phrase véhiculée par l'école :

Jules Dufilet avait bien du mal à ouvrir la bouteille.

Avec son couteau, il ôta la cire rouge qui recouvrait le bouchon.

Un bouchon de liège très abîmé ! Il le retira... et que trouva-t-il

à l'intérieur de la bouteille? Un génie ? Pas du tout !

Une feuille de papier enroulée, une feuille jaunie et très ancienne.

"Oh, oh ! dit le pêcheur, surpris. Sapristi, on dirait une carte...

- Une carte au trésor, papi ?"

Doc. 1. Drôle de pêche, Ann Rocard, p. 5.

La phrase n'est en fait qu'une «unité pratique» (Béguelin, 2002; Berrendonner \& Béguelin, 1989) qui n'a pas de statut théorique, mais sans doute «pratique» (Deulofeu, 1991). Ce savoir pratique se répercute même sur la langue parlée puisque les enseignants demandent aux élèves de produire des phrases à l'oral, comme si l'on devait parler comme l'on écrit (Campana, 2002, p. 64). Il en résulte que les élèves se représentent la langue orale à travers le prisme de l'écrit (ils disent faire des fautes en parlant) et y transposent la notion de «phrase» et de «mot» (Roubaud, 2014). Or même si les élèves parlent de «phrase», cela ne signifie pas qu'ils ont construit la notion, car cet exercice relève d'un long savoir-faire, comme le déclare Blanche-Benveniste (1999) dans un manuscrit :

Pas moyen d'échapper à cette circularité dans le domaine des savoirs pratiques. Pour que l'on comprenne ce qu'est une phrase et ce qu'est un mot, il faut d'abord avoir accumulé des exemples prototypiques (des phrases simples, des mots sans problèmes) avant d'aborder ensuite les 
exemples atypiques comme on en voit dans les textes écrits sans ponctuation ou avec pratique des points qui segmentent les phrases. (Roubaud, 2013, p. 189)

Alors comment les jeunes enfants font-ils pour construire la notion de phrase ? Quelles représentations se font-ils de cet objet insaisissable que représente la phrase?

\subsection{Représentations des élèves sur la phrase}

Pour approcher les représentations des élèves sur la phrase, nous nous appuyons sur trois expérimentations qui donnent à voir certains éléments qui peuvent «faire une phrase» pour des élèves de 6 à 8 ans.

L'une (référencée E1 dans les exemples) porte sur les savoirs déclaratifs de 26 élèves de 6-7 ans de deux classes différentes en juin 2010, confrontés à cinq énoncés ponctués. Lors de cette enquête, l'expérimentatrice demandait à chacun des élèves : «Est-ce que c'est une phrase?» et «Pourquoi dis-tu que c'est (ce n'est pas) une phrase?»; les réponses étaient enregistrées. L'objectif était de mesurer le savoir des élèves sur la phrase ainsi que sur le verbe en fin d'année (Gomila \& Roubaud, 2013). Les énoncés soumis (qui seront notés entre crochets dans les exemples relevés) ${ }^{3}$ avaient été choisis en fonction du lexème verbal : perception de la marque du pluriel «ent», identification d'un verbe d'état (être), difficultés liées à la nominalisation d'un verbe (bousculade), à la séquence verbale (modal aller suivi d'un infinitif) et à l'absence du sujet (cas de l'impératif).

Les deux autres expérimentations concernent les connaissances procédurales ${ }^{4}$ d'élèves de 7-8 ans en situation de devoir ponctuer un texte narratif (inventé pour la recherche); les justifications des élèves ont été enregistrées. Dans l'expérimentation de Caddéo (1998), le texte comprenait quatre passages au discours direct et 18 enfants de 7-8 ans (de différentes classes), par groupes de 3 ou 4, ont effectué la tâche. Dans celle de Lemaître (1999), le texte bref, avec un passage au discours direct, a été ponctué par chacun des 49 élèves de 7-8 ans (de 7 classes différentes).

3. Les cinq énoncés choisis étaient : Les enfants jouent dans la cour. La petite fille est malade. Pas de bousculade dans les couloirs. Nous allons marcher dans la colline. Regarde ce livre.

4. Les savoirs déclaratifs s'expriment «dans le langage» et les connaissances procédurales s'évaluent «dans l'activité finalisée» (George, 1988, p. 104). 
Les résultats de ces trois enquêtes montrent que, conformément aux prescriptions ministérielles $(B O, 2008$, p. 32) et à l'enseignement reçu, les élèves s'appuient sur le point et la majuscule pour identifier une phrase mais tous ne lui accordent pas la même importance. Pour certains élèves, c'est le point qui est repéré en premier. Pour d'autres, c'est la majuscule mais ce n'est pas la simple écriture d'une lettre en majuscule qui est mise en avant, c'est le rôle joué par cette lettre :

Maud : la phrase se finit par un point et commence par une majuscule (E1)

Romain : c'est une phrase parce qu'il y a LA ${ }^{5}$ majuscule et le point (E1)

Deux autres indices visuels sont mentionnés par les jeunes élèves : la longueur et les «petits mots» qui permettent d'identifier une phrase.

L'unité phrase ne doit pas être trop petite (Caddéo, 1998, p. 268), mais cette subjectivité est difficilement mesurable. Ainsi pour la phrase «Regarde ce livre», certains élèves la jugent assez longue alors que d'autres refusent de la qualifier de phrase :

Appoline : c'est une phrase mais une petite (E1)

Tom : une phrase c'est long il y a plusieurs mots + [Regarde ce livre.] c'est pas une phrase + il n'y a pas assez de mots (E1)

Se basant sur ce critère de longueur, les élèves jeunes font correspondre la ligne à la phrase, si bien que lorsque l'enseignant leur demande de compter les phrases, bon nombre d'élèves comptent les lignes. En situation de ponctuer un texte, ils utilisent la même démarche et se servent du passage à la ligne pour ponctuer (Caddéo, 1998, p. 261).

Pour identifier une phrase, les élèves utilisent un autre indice visuel; ils repèrent ce qu'ils appellent des «petits mots» comme l'indiquent ces deux commentaires sur la phrase «Pas de bousculade dans les couloirs.» :

Abderhamane : c'est une phrase + dans une phrase il y a plein de petits mots dans une phrase (E1)

Julie : c'est une phrase + elle est pas longue + il y a pas beaucoup de mots + mais surtout il y a des petits mots + dans + les + de + pas (E1)

Ces petits mots correspondent généralement à une catégorie grammaticale particulière puisque ce sont les pronoms et les déterminants qui, au moment de ponctuer un texte, servent de repère pour la majuscule,

5. Les capitales indiquent une syllabe accentuée par l'élève, ici le mot «la». 
l'école favorisant ces modèles de phrases, comme le relève Lemaître (1999) :

Les exercices scolaires, qui présentent souvent des phrases canoniques simples (article + nom (ou pronom) + verbe + complément) peuvent contribuer à la mise en mémoire de mots jugés comme étant immanquablement des initiales de phrases. (p. 12-13)

Ce que les élèves recherchent, ce ne sont pas des éléments grammaticaux mais «un certain type de lexique formant le paradigme des morphèmes grammaticaux » (Caddéo, 1998, p. 264). Ce qu'ils construisent, c'est une «catégorie de savoir commun» (Gomila, 2013, p. 151), bien utile lorsqu'ils veulent segmenter un texte, d'où des découpages bizarres au moment de ponctuer :

Ex. 21 : et caramel approche de anne qui. Le prend dans ses bras (Caddéo)

Le critère sémantique, tel que la grammaire d'Arrivé, Gadet et Galmiche (1986, p. 529) l'avance, est quelquefois mis en avant par les élèves qui font appel à la complétude sémantique pour repérer une phrase. Et dans ce cas, les points peuvent servir à délimiter des unités de sens (ou «unités d'information», Lemaître, 1999, p. 16) :

Emmanuel : j'ai mis un point parce que ça parle pas du même sujet (Lemaître)

Cécile : j'ai mis un point là [...] parce que là [avant le point] il parle d'un truc et là [après le point] il parle d'un autre truc (Lemaître)

En revanche, le critère syntaxique repéré dans la grammaire de Riegel, Pellat et Rioul (1994, p. 104) n'est mentionné que très rarement. Un nombre infime d'élèves a l'intuition d'une dépendance syntaxique entre les éléments, sans avoir les mots pour le dire :

Thomas : [Nous allons marcher dans la colline.] c'est une phrase il y a un pronom et un verbe (E1)

Les représentations des élèves révèlent des procédures particulières pour identifier une phrase, procédures insoupçonnées le plus souvent des enseignants. Ces savoirs sur la phrase se construisent à partir de bribes d'informations : savoirs enseignés, remarques ponctuelles de l'enseignant à l'occasion d'activités de lecture ou d'écriture, mises en mémoire de certaines occurrences rencontrées. Comme l'écrit Caddéo (1998) : «Il est vrai que les modèles de phrases sont cherchés dans les 
modèles scolaires mais ils sont également réorganisés à partir d'une intuition linguistique personnelle.» (p. 268)

Une façon de débusquer cette intuition linguistique est lorsque les enfants s'apprennent à écrire, comme le pensent Boré et Bosredon (2013) : «[...] c'est l'activité d'écriture prise comme long processus verbal extériorisé et socialisé qui révèle et modifie les idées des élèves à propos de la phrase et du texte.» (p.15) Nous considérons, à la suite de ces auteurs, que les apprentis-scripteurs, répondant à une tâche d'écriture (écrire une suite de récit, écrire une lettre, décrire un personnage, etc.), produisent des textes, quel qu'en soit le degré de cohérence. Nous allons maintenant réexaminer la notion de phrase au travers de quelques productions.

\section{La phrase dans les textes d'élèves (5-8 ans)}

Nous avons recueilli auprès des enseignants, depuis les années 1990, des textes d'élèves obtenus non dans des conditions expérimentales, mais attestés par des pratiques ordinaires. Pour la tranche d'âge qui nous intéresse, le corpus compte 690 textes dont un petit nombre écrit par des enfants de 5-6 ans ${ }^{6}$ et qui sont dans $86 \%$ des cas des productions narratives. Comme le déclarent Elalouf et Boré (2007), «il existe une répartition des genres scolaires dans l'école et le collège» (p. 63). Notre base de données comprend des textes manuscrits, écrits individuellement ainsi que les situations d'écriture qui les ont engendrés (consignes, documents fournis aux élèves...).

Notre démarche est inductive et exploratoire : elle vise à révéler des textes, à en explorer les régularités, à rassembler les traits linguistiques qui ont tendance à apparaitre ensemble. Ces écrits sont utilisés comme un matériau pour comprendre la grammaire propre de l'élève et non comme un réservoir d'erreurs (Cappeau \& Roubaud, 2005). En effet, les textes donnent à voir les usages que les enfants font de la langue. Lors de la constitution de cette base de données ${ }^{7}$, nous avions ainsi repéré, chez les enfants de 5 à 8 ans, des façons de faire récurrentes pour découper leur texte; c'est ce que nous allons présenter en prenant appui

6. En France, les élèves apprennent à lire et à écrire des textes à 6 ans, d'où le faible nombre de productions d'élèves de 5 à 6 ans.

7. Elle comprend actuellement 1600 textes de 5 à 11 ans, en cours d'informatisation, ce qui va permettre de quantifier les phénomènes repérés. 
sur quelques productions écrites, choisies pour leur degré de représentativité des façons de segmenter.

Cette analyse qualitative s'inscrit dans le cadre fourni par l'Approche pronominale (Blanche-Benveniste, Deulofeu, Stéfanini \& Eynde, 1984) avec la méthodologie du Groupe aixois de recherches en syntaxe (GARS) qui renonce à faire de la phrase une unité centrale pour la description syntaxique et se fonde sur la notion de construction syntaxique qu'il est possible de définir sur une base formelle (Deulofeu, 1991; Rossi-Gensane, 2010; Sabio, 2006). Cette option méthodologique, qui libère de la pression de la phrase graphique véhiculée par l'école, amène à découvrir des régularités dans le découpage de textes, faisant écho aux représentations des élèves (cf. 3.1). Mais au-delà, les productions d'élèves jeunes laissent entrevoir des conduites discursives qui dépassent le cadre de la phrase graphique (cf. 3.2).

\subsection{Des représentations aux productions}

Dans les textes qui vont suivre ${ }^{8}$, écrits par des enfants jeunes, nous allons analyser les repères évoqués précédemment par les élèves en situation d'identifier une phrase ou de délimiter un texte en phrases (cf. supra). Notre objectif est de voir la façon dont ils utilisent ces repères en production ${ }^{9}$.

\subsubsection{L'usage de la ligne}

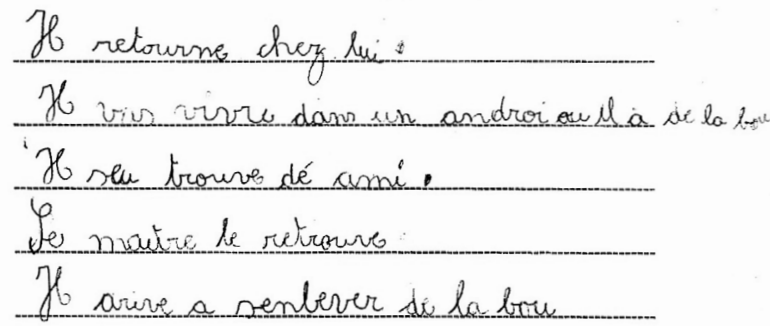

Texte 1 : Charlotte, 7-8 ans (décembre).

8. La transcription orthographique des textes sélectionnés figure en annexe.

9. Les textes sélectionnés sont représentatifs d'autres textes du corpus pour lesquels nous aurions pu faire la même analyse mais que nous ne pouvons pas faire figurer ici, faute de place. 
L'élève devait écrire la suite de l'histoire de Porculus (texte 1), petit cochon désireux de vivre dans la boue de la ferme, d'où l'emploi du pronom anaphorique «il». Conformément aux représentations des élèves, chaque phrase occupe une ligne. Cette prégnance de l'équation «une phrase = une ligne» est telle que l'élève dépasse la ligne de la feuille pour achever l'écriture de sa deuxième phrase (Il vas vivre dans un androi ou il a de la bou).

Mais il est à remarquer que chaque ligne correspond à une construction verbale dont chacune commence par une majuscule mais ne finit pas obligatoirement par un point. La ligne semble tenir un rôle plus important que la ponctuation car elle est le support matériel des différentes constructions syntaxiques. Ce qui est en jeu ici, ce n'est pas la phrase graphique mais la phrase en tant qu'unité de syntaxe ${ }^{10}$, unité qui possède une organisation syntaxique satisfaisante (généralement complète) et qui trouve dans la ligne un support à sa matérialisation.

\subsubsection{Le recours aux connecteurs}

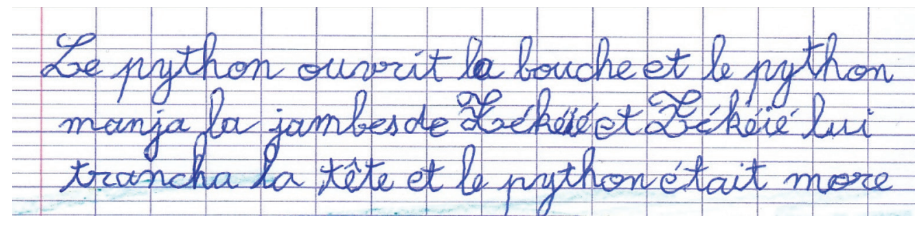

Texte 2 : Clara, 6-7 ans (mars).

L'élève devait écrire la suite d'un récit lu par l'enseignante : l'histoire d'un petit garçon Zékéyé face à un python (texte 2). Aucune ponctuation n'apparait, hormis la majuscule de début de texte. Même si cette production ne comporte aucune phrase graphique, il reste toutefois possible d'identifier des constructions syntaxiques : quatre constructions verbales toutes reliées par le connecteur $e t^{11}$.

La présence des répétitions sur la place sujet (le python) montre que l'élève progresse «pas à pas» dans son récit (Schneuwly, 1988) en appliquant à l'écrit le système de l'oral : d'une gestion locale "pas à pas» vers une gestion globale. Employer des connecteurs de l'oral est

10. Paolacci et Rossi-Gensane (2014) emploient le terme de «phrase syntaxique».

11. Catach (1994) a montré que les conjonctions servaient en ancien français à rythmer et à structurer le récit et qu'elles ont été progressivement remplacées par la ponctuation. 
un moyen de segmenter une production, l'absence de marque de ponctuation étant compensée parfois par la multiplication de connecteurs (Roubaud \& Garcia-Debanc, 2014, p. 320).

\subsubsection{Les mots "accentués " (porteurs de la majuscule)}

Mardi 7 mars
Genvente la suite de l' histoire.

Pendent que la chève était anfermer

La chàve casé la corde et ocies la porte

Bt envivire pendent que monsieur seguir

R gardas la télé la chè vere etact doga

Gano la montagine et monsieu segéin

Ale arve pas que blenquatte sechapet.

et blencette so lateavec le but

lout la muit eltituen nquette ans perdu

Texte 3 : Anthony, 7-8 ans (mars).

Dans cette situation d'écriture de la suite du conte La chèvre de Monsieur Seguin (texte 3), la majuscule est utilisée systématiquement en début de ligne, sauf pour les deux dernières lignes. En conséquence, la segmentation du texte par les majuscules coupe des constructions verbales : le verbe (Regardes) n'est plus rattaché à son sujet (monsieur seguin) et la négation $(\mathrm{Ne}$ ) sépare le sujet (monsieu segein) du verbe save $^{12}$, entrainant ainsi une incomplétude sémantique et syntaxique de ces constructions syntaxiques.

12. Ce n'est qu'à l'impératif que le verbe et la négation portent la majuscule : Regarde la télé. Ne viens pas. 
On peut remarquer que différents critères sont mobilisés pour délimiter une phrase : la place initiale des mots accentués (en début de ligne), le recours à des morphèmes grammaticaux courts, candidats susceptibles de porter une majuscule : les pronoms («je» de Ginvinte de la consigne), les déterminants ( $L a)$, les prépositions (Dans), les conjonctions $(E t)$, les adverbes de négation $(\mathrm{Ne})$. D'autres textes du corpus montrent que les pronoms sujets ( $\mathrm{il}$ ou elle) sont des candidats favoris à la majuscule lors de l'écriture, ce qui n'est pas étonnant puisque ces pronoms sont souvent accentués dans les récits lus en classe ou dans les exercices scolaires. Les jeunes scripteurs structurent leur texte avec les repérages qu'ils ont faits.

\subsubsection{L'appui sur le genre}

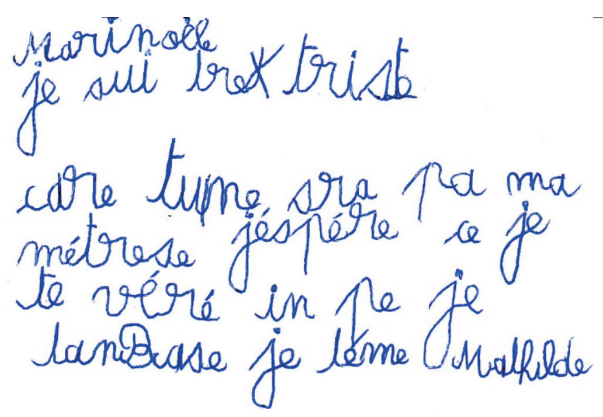

Texte 4 : Mathilde, 5-6 ans (juin).

Dans cette situation d'écriture d'un texte libre (texte 4) par une élève très jeune (en fin d'école maternelle), aucune ponctuation n'apparait. Or ce texte est tout à fait lisible, moyennant une lente subvocalisation, comme lors de la lecture à voix haute des textes anciens écrits sans ponctuation. Dans de telles productions, la notion de phrase graphique devient inopérante au profit de celle de construction syntaxique.

La réception que nous avons de ce texte est liée au genre : les indices inhérents à la lettre (mise en espace dans la feuille, mention du je de l'expéditeur et du $t u$ du destinataire, formule de politesse) aident à la lecture. Dès 4 ans, les enfants différencient les genres les plus connus (Chanquoy \& Fayol, 1995; Teberosky, 1993). S'appuyer sur le genre pour écrire est une aide pour l'élève au moment de segmenter son texte.

\subsubsection{Synthèse}

L'analyse de ces quelques textes fait écho aux représentations des jeunes scripteurs sur la phrase à l'écrit : ces dernières avaient mis en 
avant la place accordée à la majuscule, le rôle joué par la ligne ainsi que le repérage d'un certain type de mots accentués (cf. 2.2).

Ce que nous avons observé dans les productions, c'est la prédominance des unités syntaxiques sur les unités graphiques. Rares sont les textes d'enfants jeunes qui comprennent à la fois des majuscules et des points. Le recours aux connecteurs pour segmenter une production est très fréquent chez les jeunes scripteurs. L'appui sur le genre est également un bon moyen pour mettre le texte en espace, sans recourir à la ponctuation. Pour certains élèves, la ligne sert de support aux constructions syntaxiques. Les morphèmes accentués sont mis en mémoire et certains scripteurs associent place du mot accentué et début de ligne.

Mais au-delà des unités syntaxiques, certains élèves traitent des unités textuelles, comme nous allons le voir au travers de l'échantillon présenté.

\subsection{Au-delà des représentations : des conduites discursives}

Dans les textes qui suivent, il est possible de repérer des conduites discursives qui dépassent le cadre des unités syntaxiques.

\subsubsection{Le point et les étapes du récit}

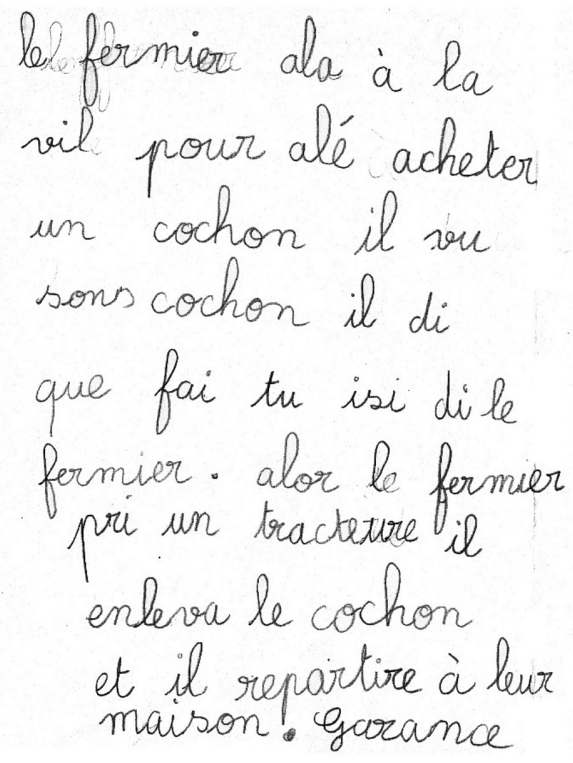

Texte 5 : Garance, 6-7 ans (juin). 
Dans cette suite du récit de Porculus (texte 5), seuls deux points apparaissent sans que l'on puisse noter la présence d'une seule majuscule. Le premier point n'a pas qu'un rôle démarcatif, il a aussi un rôle énonciatif : il sert à marquer la frontière des épisodes dans les narrations, ce qui est fréquent au début de l'apprentissage de l'écrit (Fayol, 1989, p. 23).

On constate chez ce jeune scripteur l'émergence de ce qui constitue un texte : la trame narrative. En effet, le point central coupe le texte en deux épisodes narratifs : celui de la recherche du cochon puis celui de la délivrance du cochon, comme si ce point avait une valeur métrique ou rythmique (Fabre, 1989, p. 65). En revanche, le dernier point clôt le texte. Les deux points sont donc à analyser comme deux unités distinctes qui possèdent chacune une valeur particulière : le système utilisé par l'enfant est donc légèrement plus riche et plus complexe que celui qui préconise qu'un point termine une phrase.

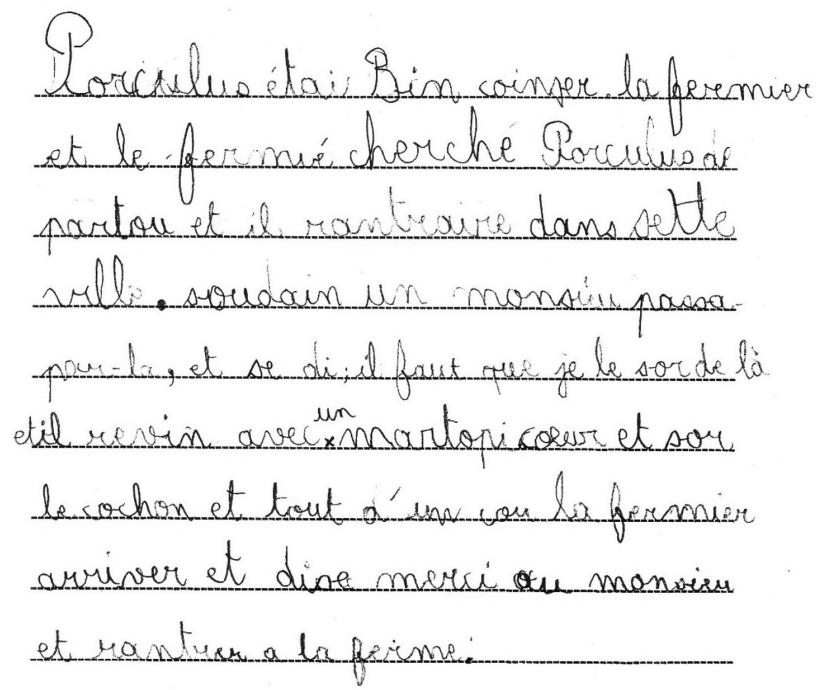

Texte 6 : Damien, 7-8 ans (décembre).

C'est la même situation d'écriture que pour le texte précédent mais la production (texte 6) est celle d'un élève plus âgé (7-8 ans). On y retrouve l'utilisation de points pour délimiter les étapes du récit mais avec la présence de trois points. Le premier point, après la première construction verbale (Porculus étai Bin coinser.), marque la situation initiale : le petit cochon est pris au piège. Le point médian sépare les 
deux épisodes narratifs (premier épisode : Porculus est prisonnier; deuxième épisode : Porculus va être délivré) et le point final clôt le récit. À l'intérieur de ces deux épisodes, le connecteur et lie les constructions syntaxiques.

On remarque dans ce texte la fréquence des constructions verbales avec mise en facteur commun du sujet : le syntagme nominal «la fermière» est le sujet de trois constructions verbales (nous corrigeons l'orthographe) : tout d'un coup la fermière arrivait et disait merci au monsieur et rentrait à la ferme. Au-delà de la coordination, la mise en facteur commun du sujet fait partie de la représentation que les élèves, quel que soit leur âge, se font de la langue écrite (Roubaud, 2014), ce qui donne un ton cérémonieux au récit dans les énumérations des actions du personnage. Il ne s'agit donc pas d'une simple coordination, mais d'un procédé de langue élaborée avec la mise en facteur commun du sujet et l'emploi du coordonnant $e t$.

Ce que ces deux textes ( 5 et 6 ) montrent, c'est une organisation du texte en périodes (au sens de Berrendonner, 2002), chacune d'elles constituant une unité d'information.

\subsubsection{Le point et le cas des épexégèses}

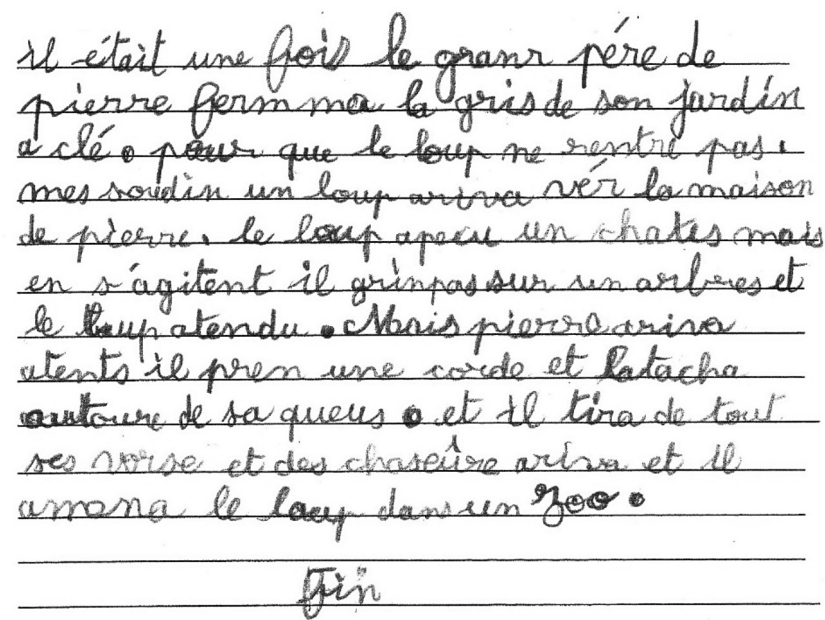

Texte 7 : Anthony, 7-8 ans (avril).

Là aussi (texte 7), les points rythment les épisodes de l'histoire (cf. textes 5 et 6), sauf dans cette séquence dont nous rétablissons l'orthographe : «il était une fois le grand-père de pierre ferma la grille de son 
jardin à clé. Pour que le loup ne rentre pas». La subordonnée exprimant le but en pour que, isolée entre deux points, fonctionne comme une épexégèse (Bally, 1944, p. 59), c'est-à-dire comme un ajout à la construction verbale précédente : sa réalisation est différée par rapport au reste de la construction. Au niveau discursif, l'ajout a l'avantage, comme le mentionne Combettes (2007), de «donner plus de force argumentative au constituant ainsi isolé» (p. 122).

Même si nous repérons, dans la séquence mentionnée plus haut, deux phrases graphiques, on ne peut pas ignorer l'existence d'un regroupement sémantique. «L'absence d'homothétie entre la syntaxe et la ponctuation» (Matthey, 2003, p. 54) peut donc cacher des effets stylistiques; ce que confirme Béguelin (2000) : «Bien des structures stigmatisées chez des élèves [...] se retrouvent toutefois, comme procédés stylistiques valorisés, sous la plume des écrivains.» (p. 268)

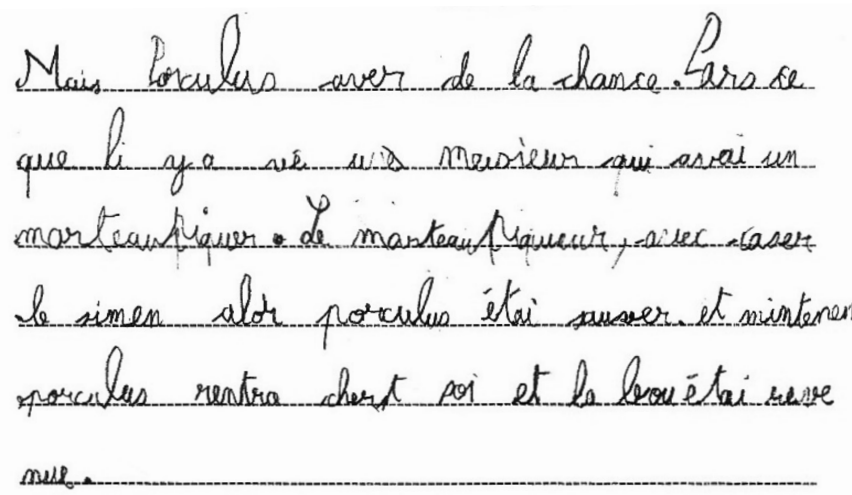

Texte 8 : Pablo, 7-8 ans (décembre).

Là encore (texte 8), les points rythment les épisodes du récit (cf. textes 5, 6 et 7). La délimitation de la première construction syntaxique correspond à une sorte de commentaire sur la suite de l'histoire que l'élève va développer. En revanche, la causale (dont nous rétablissons l'orthographe) «Parce que il y a un monsieur qui avait un marteaupiqueur.» fonctionne comme une épexégèse et cet effet stylistique met en évidence le motif de la chance de Porculus.

Dans les textes d'élèves plus âgés, nous avons également relevé des épexégèses dont un certain nombre à valeur temporelle comme dans l'exemple suivant, produit par un élève de 9 ans : Quoi elle était bien trenquil. Lundi soir. 
Une recherche ultérieure devrait permettre un recensement de ces segments isolés entre deux points et rattachés sémantiquement à la construction précédente. Dresser une liste de ces compléments différés aiderait à mieux comprendre les regroupements sémantiques que les élèves privilégient.

\subsubsection{Le regroupement d'unités énonciatives}

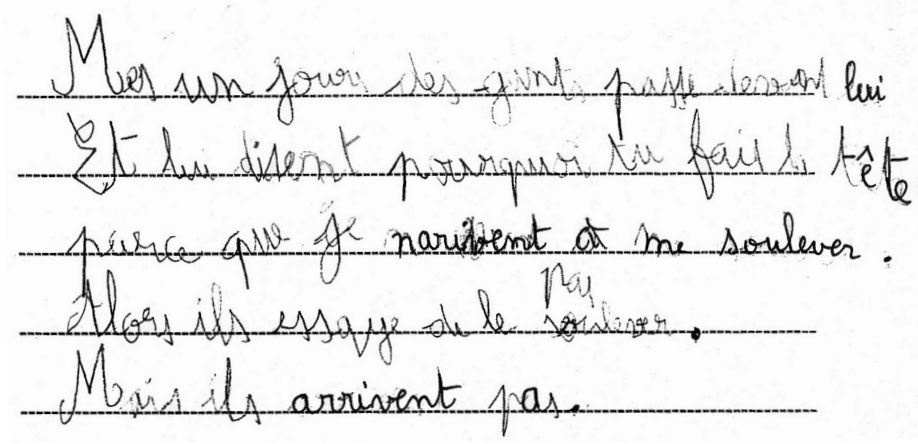

Texte 9 : Paul, 7-8 ans (décembre).

Dans cette suite de récit de Porculus (texte 9), nous retrouvons le rôle de la ligne, porteuse de la construction verbale et son dépassement (cf. texte 1) ainsi que la prégnance de la majuscule en début de phrase qui correspond au commencement de la ligne (cf. textes 1 et 3 ). Un seul mot fait exception (parce) alors qu'il est placé en début de ligne. Or la construction verbale, dont nous rétablissons l'orthographe, «parce que je n'arrive pas à me soulever» est une réponse au pourquoi de la construction verbale précédente «Et lui disent pourquoi tu fais la tête».

La non-présence de la majuscule sur le mot parce pourrait indiquer une prise en compte par l'élève d'une «unité énonciative» qui dépasse le simple cadre de la phrase graphique. Comme l'écrit BlancheBenveniste (2002) :

Dans les objets qu'on appelle phrases, se trouvent habituellement mêlées des dépendances hétérogènes, les unes se référant au verbe et les autres non, avec des propriétés syntaxiques très différentes. Le terme unique de phrase fait croire que les relations sont d'une seule espèce. (p. 20)

Comme pour les épexégèses, il faudrait poursuivre l'étude afin de lister quels types de regroupements d'unités les élèves acceptent ou refusent, les questions-réponses semblant déjà en faire partie. 


\subsubsection{Synthèse}

L'observation de ces quelques textes nous amène à distinguer, pour certains élèves, un niveau de découpage supérieur à celui des unités syntaxiques : celui des unités textuelles.

Le point est alors un bon outil pour ce découpage et a plusieurs fonctions. Il peut marquer des périodes, des «unités discrètes» (Fabre, 1989) correspondant à des épisodes narratifs. Il permet de différer des compléments, créant ainsi un effet stylistique. Il sert à encadrer des regroupements sémantiques, comme les questions-réponses.

On constate ainsi que les regroupements que les jeunes scripteurs opèrent sont des traces d'organisation textuelle et non pas des marques de défaillance syntaxique.

\section{Conclusion}

Nous devons éviter d'observer les signes de ponctuation utilisés par les enfants en les liant uniquement aux emplois que nous leur connaissons. Si connaitre les représentations des élèves est utile à l'enseignant (Lemaître, 1999) et au futur enseignant (Paolacci \& Garcia-Debanc, 2005) afin qu'il élargisse sa propre conception de la phrase (Rondelli, 2013, p. 80), cela n'est pas suffisant.

L'analyse des textes scolaires montre qu'au-delà de la mise en application des indices repérés pour définir une phrase, les élèves utilisent des stratégies de segmentation qui dépassent le simple cadre de la phrase graphique. Ils restituent à l'écrit des unités syntaxiques et certains scripteurs prennent en compte des unités textuelles qui éclairent et renforcent l'analyse que l'on peut faire des constructions verbales.

Il est nécessaire de poursuivre l'inventaire et la description des unités énonciatives, qui ne sont pas dans les inventaires scolaires, si l'on veut saisir le fonctionnement des élèves et par là même leurs attentes.

Si l'enseignant en reste à la conception scolaire de la phrase, il lui sera difficile de percevoir toute la richesse des organisations dans les textes de ses élèves.

\section{RÉFÉRENCES BIBLIOGRAPHIQUES}

Arrivé, Michel, Gadet, Françoise \& Galmiche, Michel. (1986). La grammaire d'aujourd'hui : guide alphabétique de linguistique française. Paris : Flammarion. 
Avanzi, Mathieu. (2007). Regards croisés sur la notion de macro-syntaxe. Travaux neuchâtelois de linguistique, 47, 39-58.

BALly, Charles. (1944). Linguistique générale et linguistique française ( $2^{\mathrm{e}}$ éd.). Berne : Francke.

Béguelin, Marie-José. (2000). De la phrase aux énoncés : grammaire scolaire et descriptions linguistiques. Bruxelles : De Boeck-Duculot.

BÉGuelin, Marie-José. (2002). Clause, période ou autre? La phrase graphique et la question des niveaux d'analyse. Verbum, 24(1-2), 85-107.

Berrendonner, Alain. (2002). Les deux syntaxes. Verbum, 24(1-2), 23-35.

Berrendonner, Alain \& Béguelin, Marie-José. (1989). Décalages. Les niveaux de l'analyse linguistique. Langue française, 81, 99-125.

Blanche-Benveniste, Claire. (1993). Faire des phrases. Travaux neuchâtelois de linguistique, 58, 221-229.

Blanche-Benveniste, Claire. (2002). Phrase et construction verbale. Verbum, 24(1-2), 7-22.

Blanche-Benveniste, Claire, Deulofeu, José, Stéfanini, Jean \& Eynde, Karel VAN DEN. (1984). Pronom et syntaxe. L'Approche pronominale et son application au français. Paris : SELAF.

Blanche-Benveniste, Claire, Bilger, Mireille, Rouget, Christine \& Eynde, Karel vAN DEN. (1990). Le français parlé. Études grammaticales. Paris : CNRS Éditions.

Boré, Catherine \& Bosredon, Catherine. (2013). La phrase selon les brouillons : un trajet entre l'oral et l'écrit. Le français aujourd'hui, 181, 13-24.

CAddÉo, Sandrine. (1998). L'usage de la ponctuation chez les enfants. Dans J.-M. Defays, L. Rosier \& F. Tikin (dir.), Actes du colloque international et interdisciplinaire de Liège : À qui appartient la ponctuation? (p. 255-273). Bruxelles : De Boeck \& Larcier.

CAMPANA, Marc. (2002). Une grammaire pour mieux écrire. Collège. Créteil : CRDP Académie de Créteil.

Cappeau, Paul \& Roubaud, Marie-Noëlle. (2005). Enseigner les outils de la langue avec les productions d'élèves. Paris : Bordas.

CATACH, Nina. (1994). La ponctuation : histoire et système. Paris : Presses universitaires de France.

Chanquoy, Lucile \& Fayol, Michel. (1995). Analyse de l'évolution et de l'utilisation de la ponctuation et des connecteurs, dans deux types de texte. Étude longitudinale du CP au CE2. Enfance, 48(2), 227-241.

ChIss, Jean-Louis \& DAvid, Jacques. (2011). Et la grammaire de phrase? Le français aujourd'hui, 5, 175-181.

Combettes, Bernard. (2007). Discontinuité et cohérence discursive : le cas des ajouts après le point. Cahiers de praxématique, 48, 111-134. 
Deulofeu, José. (1991). La notion de dépendance syntaxique dans l'approche pronominale. L'information grammaticale, 50, 19-23.

Dubois, Jean \& Lagane, René. (1973). La nouvelle grammaire du français. Paris : Larousse.

Elalouf, Marie-Laure \& Boré, Catherine. (2007). Construction et exploitation de corpus d'écrits scolaires. Revue française de linguistique appliquée, 12(1), 53-70.

FABre, Claudine. (1989). Les débuts de la ponctuation au CP. Études de linguistique appliquée, 73, 59-69.

FAYOL, Michel. (1989). Une approche psycholinguistique de la ponctuation. Étude en production et compréhension. Langue française, 81, 21-39.

George, Christian. (1988). Interaction entre les connaissances déclaratives et procédurales. Dans P. Perruchet (dir.), Les automatismes cognitifs (p. 103-137). Liège : Mardaga.

Gomila, Corinne. (2009). Premières interventions pratiquées lors de l'enseignement de la lecture au Cours préparatoire (CP). Dans J. Dolz \& C. Simard (dir.), Pratiques d'enseignement grammatical. Points de vue de l'enseignant et de l'élève (p. 75-97). Laval : Presses de l'Université Laval.

Gomila, Corinne. (2013). Le Petit mot de la classe : entre catégorisation pratique et classification grammaticale. Dans O. Bertrand \& I. Schaffner (dir.), Enseigner la grammaire (p. 145-158). Palaiseau : Éditions de l'École polytechnique.

Gomila, Corinne \& Roubaud, Marie-Noëlle. (2013). Le verbe au cours préparatoire : premières constructions du concept. Dans C. Avezard-Roger \& B. Lavieu-Gwozdz (dir.), Le verbe : perspectives linguistiques et didactiques (p. 31-45). Arras : Artois Presses Université.

LEMAîTRE, Brigitte. (1999). Comment faire pour ponctuer un texte? Représentations et procédures d'élèves de CE1. Spirale, 23, 5-20.

Matthey, Marinette. (2003). Micro et macrosyntaxe : chimères de linguistes ou notions utiles pour la didactique? Le français aujourd'hui, $141,52-58$.

Ministère de L'Éducation nationale et Ministère de L'Enseignement SUPÉRIEUR ET DE LA RECHERCHE. (2008). BO. Horaires et programmes d'enseignement de l'école primaire. Hors-série n 3 du 19 juin 2008.

Paolacci, Véronique \& Garcia-Debanc, Claudine. (2005). Comment former à l'enseignement de la ponctuation? Analyse de pratiques effectives de formation initiale. Pratiques, 125-126, 85-114.

Paolacci, Véronique \& Rossi-Gensane, Nathalie. (2014). Ponctuation et écrits d'élèves : une conception différente de la phrase pour enseigner la ponctuation autrement. Le français aujourd'hui, 187, 115-125. 
Riegel, Martin, Pellat, Jean-Christophe \& Rioul, René. (1994). Grammaire méthodique du français. Paris : Presses universitaires de France.

Rondelli, Fabienne. (2013). La phrase, segment textuel «de base»? Choix d'écriture d'élèves de cycle 3 et jugements des enseignants. Le français aujourd'hui, 181, 71-81.

Rossi-Gensane, Nathalie. (2010). Encore quelques remarques sur la phrase. La linguistique, 46(2), 69-107.

Roubaud, Marie-Noëlle. (2013). Langue et enseignement. Une sélection de 22 manuscrits de Claire Blanche-Benveniste (de 1976 à 2008). Neuchâtel : Travaux neuchâtelois de linguistique.

Roubaud, Marie-Noëlle. (2014). De la description de langue à son enseignement (Mémoire d'habilitation à diriger des recherches). Université Stendhal-Grenoble 3, Grenoble.

Roubaud, Marie-Noëlle \& Garcia-Debanc, Claudine. (2014). L'approche d'«anomalies» dans des textes narratifs d'élèves de fin d'école primaire (10-11 ans). Quelques pistes pour la lecture des textes par les enseignants. Dans M. Avanzi, V. Conti, G. Corminboeuf, F. Gachet, L. A. Johnsen \& P. Montchaud (dir.), Enseignement du français : les apports de la recherche en linguistique. Réflexions en l'honneur de Marie-José Béguelin (p. 307-325). Bruxelles : Peter Lang.

SABIo, Frédéric. (2006). Phrases et constructions verbales : quelques remarques sur les unités syntaxiques dans le français parlé. Dans D. Lebaud, C. Paulin \& K. Ploog (dir.), Constructions verbales et production de sens (p. 127-139). Besançon : Presses universitaires de FrancheComté.

Schneuwly, Bernard. (1988). Le langage écrit chez l'enfant. Paris : Delachaux et Niestlé.

Seguin, Pierre. (1993). L'invention de la phrase au XvIII siècle : contribution à l'histoire du sentiment linguistique français. Louvain : Peeters.

Teberosky, Ana. (1993). L'écrit chez les pré-scolaires. Le français dans le monde, $\mathrm{n}^{\circ}$ spécial, 88-107. 


\section{ANNEXE. - Transcription orthographique des textes d'élèves (ponctuation, majuscules et mise en page conservées)}

\begin{tabular}{|c|c|}
\hline Textes présentés en 3.1 & Textes présentés en 3.2 \\
\hline $\begin{array}{l}\text { Texte } 1 \text { : Charlotte ( } 7-8 \text { ans, décembre) } \\
\text { Il retourne chez lui. } \\
\text { Il va vivre dans un endroit où il a de la boue } \\
\text { Il s'est trouvé des amis. } \\
\text { Son maitre le retrouve } \\
\text { Il arrive à s'enlever de la boue }\end{array}$ & $\begin{array}{l}\text { Texte } 5 \text { : Garance (6-7 ans, juin) } \\
\text { le fermier alla à la } \\
\text { ville pour aller acheter } \\
\text { un cochon il vit } \\
\text { son cochon il dit } \\
\text { que fais-tu ici dit le } \\
\text { fermier. alors le fermier } \\
\text { prit un tracteur il } \\
\text { enleva le cochon } \\
\text { et ils repartirent à leur } \\
\text { maison. Garance }\end{array}$ \\
\hline $\begin{array}{l}\text { Texte } 2 \text { : Clara (6-7 ans, mars) } \\
\text { Le python ouvrit la bouche et le python } \\
\text { mangea la jambe de Zékéyé et Zékéyé lui } \\
\text { trancha la tête et le python était mort }\end{array}$ & $\begin{array}{l}\text { Texte } 6 \text { : Damien (7-8 ans, décembre) } \\
\text { Porculus était Bien coincé. la fermière } \\
\text { et le fermier cherchaient Porculus de } \\
\text { partout et ils rentrèrent dans cette } \\
\text { ville. soudain un monsieur passa } \\
\text { par là, et se dit, il faut que je le sorte de là } \\
\text { et il revint avec un marteau-piqueur et sort } \\
\text { le cochon et tout d'un coup la fermière } \\
\text { arrivait et disait merci au monsieur } \\
\text { et rentrait à la ferme. }\end{array}$ \\
\hline $\begin{array}{l}\text { Texte } 3 \text { : Anthony (7-8 ans, mars) } \\
\text { J'invente la suite de l'histoire. } \\
\text { Pendant que la chèvre était enfermée } \\
\text { La chèvre cassait la corde et aussi la porte } \\
\text { Et enfuit pendant que monsieur seguin } \\
\text { Regarde la télé la chèvre était déjà } \\
\text { Dans la montagne et monsieur seguin } \\
\text { Ne savait pas que blanquette s'échappait. } \\
\text { et blanquette se battait avec le loup } \\
\text { toute la nuit et blanquette avait perdu }\end{array}$ & $\begin{array}{l}\text { Texte } 7 \text { : Anthony (7-8 ans, avril) } \\
\text { il était une fois le grand-père de } \\
\text { pierre ferma la grille de son jardin } \\
\text { à clé. pour que le loup ne rentre pas. } \\
\text { mais soudain un loup arriva vers la maison } \\
\text { de pierre. le loup aperçut un chat mais } \\
\text { en s'agitant il grimpa sur un arbre et } \\
\text { le loup attendit. Mais pierre arriva } \\
\text { à temps il prend une corde et l'attacha } \\
\text { autour de sa queue. et il tira de toutes } \\
\text { ses forces et des chasseurs arrivèrent et il } \\
\text { amena le loup dans un zoo. } \\
\qquad \begin{array}{c}\text { fin }\end{array}\end{array}$ \\
\hline \multirow[t]{2}{*}{$\begin{array}{l}\text { Texte } 4 \text { : Mathilde (5-6 ans, juin) } \\
\text { Marie-Noëlle } \\
\text { je suis très triste } \\
\text { car tu ne seras pas ma } \\
\text { maitresse j'espère que je } \\
\text { te verrai un peu je } \\
\text { t'emBrasse je t'aime Mathilde }\end{array}$} & $\begin{array}{l}\text { Texte } 8 \text { : Pablo (7-8 ans, décembre) } \\
\text { Mais Porculus avait de la chance. Parce } \\
\text { que il y avait un monsieur qui avait un } \\
\text { marteau-piqueur. Le marteau-piqueur, avait cassé } \\
\text { le ciment alors porculus était sauvé. et maintenant } \\
\text { porculus rentra chez soi et la boue était reve- } \\
\text { nue. }\end{array}$ \\
\hline & $\begin{array}{l}\text { Texte } 9 \text { : Paul ( } 7-8 \text { ans, décembre) } \\
\text { Mais un jour des gens passent devant lui } \\
\text { Et lui disent pourquoi tu fais la tête } \\
\text { parce que je n'arrive pas à me soulever. } \\
\text { Alors ils essayent de le soulever. } \\
\text { Mais ils arrivent pas. }\end{array}$ \\
\hline
\end{tabular}

